\title{
Enhancement of $\mathrm{Eu}^{3+}$ emission in solution by bulky chelating ligands and deuterium substitution
}

\author{
Mohammed A. Zaitoun \\ Department of Chemistry, Mutah University, Karak, Jordan \\ E-mail:zaitoun@mutah.edu.jo
}

\begin{abstract}
Binding the europium ion with a bulky chelating ligand provides one strategy for minimizing the quenching effects of water on the luminescence of the ion. 1,4,8,11-tetraazacyclotetradecane-1,4,8,11-tetraaceticacid tetrahydrochloride (TTT) was used as a chelating agent to isolate $\mathrm{Eu}^{3+}$ from hydroxyl deactivation in water. A large enhancement in the $\mathrm{Eu}^{3+} \mathrm{emission}^{3}$ was observed for the chelated $\mathrm{Eu}^{3+}$ compared to the unchelated. Absorption and emission spectra are reported together with lifetime measurements. The latter were used to study concentration quenching and quenching by water.
\end{abstract}

\section{Introduction}

Rare earth ions have been used as phosphors and laser materials. The trivalent rare earth ions exhibit sharp and intense luminescence bands that result from $\mathrm{f}-\mathrm{f}$ electronic transitions [1]. A common problem that is encountered with the emission of rare earth ions in aqueous solution is quenching by water. Concentration quenching may also pose a problem [2]. There are two strategies to alleviate this quenching. One is to isolate the metal ion from deactivating groups (chelation) [3-5] and the other is to encapsulate (dope) the ion in a solid matrix, where the solid matrix containing the rare earth ion is then heated to high temperatures to lessen the effect of deactivating groups (hydroxyl groups). The later method lacks the advantage of low temperature processing and destroys other molecules whose presence may be desired for purposes such as energy transfer to the lanthanides [6-8].

$\mathrm{Eu}^{3+}$ is one of the few metallic aquo cations in the periodic table that luminesces in solution at room temperature [9]. The unique spectroscopic properties of $\mathrm{Eu}^{3+}$ make it as an optical probe of local structure $[1,10]$. Laser excited $\mathrm{Eu}^{3+}$ luminescence spectroscopy is a powerful tool for monitoring the binding of this ion to ligands in solution [11]. Lanthanide ions form stable crystalline complexes with heterocyclic ligands which exhibit efficient energy transfer from the absorbing coordinated ligands to the chelated lanthanide ions. These complexes can absorb UV radiation with the organic portion of the complex molecule, followed by intramolecular energy transfer to $\mathrm{Eu}^{3+}$ ions and can emit visible light at individual wavelengths with the visible region with higher efficiency than unchelated lanthanide ions [12]. Absent any accidental coincidences, each $\mathrm{Eu}^{3+}$ complex will produce a unique excitation spectrum, the intensity of which is proportional to the concentration of the complex in solution. Use of a pulsed laser as the excitation source allows the measurement of $\mathrm{Eu}^{3+}$ excited state lifetimes, $\tau$, which are characteristic of each complex [11]. 
<smiles></smiles>

Structure 1.

Complexes formed with aminopolycarboxylic ligands have shown to be particularly stable $[11,13]$. TTT (Structure 1) is an aminocarboxylic, heterocyclic, commercially available, water soluble, and multidentate ligand ( 8 chelating sites) that is expected to form a stable complex with $\mathrm{Eu}^{3+}$. TTT is sufficiently strong to effectively chelate all the $\mathrm{Eu}^{3+}$ ions. The ligand might be unable to completely surround the rare earth ion uniformly and, therefore, present an asymmetric environment. The bonding groups are not all identical, further adding to the asymmetry.

Since the $\mathrm{Eu}^{3+}$ transitions depend for their intensity largely on the degree of asymmetry in the environment. Hence the formation of a unique asymmetric environment surrounding the rare earth ion should lead to a marked enhancement of the fluorescence primarily due to the increased transition probability for fluorescence but also to the greater efficiency of pumping because of the increased absorption as the order of asymmetry of europium species. The general sharpening and resolution would be consistent with the crystal field surrounding the rare earth ion [14].

In this work we used TTT as an aminocarboxylic ligand to protect the europium ion in solution. TTT has 8 chelating sites for binding the trivalent europium ion; the enhanced emission of chelated $\mathrm{Eu}^{3+}$ emission compared to the emission of unchelated europium shows the success of the chelating strategy.

\section{Results}

\subsection{Absorption spectroscopy}

The absorption spectrum of a $1 \times 10^{-3} \mathrm{M} \mathrm{Eu}^{3+}$ with excess-TTT in water is shown in Fig. 1. Resolved peaks or shoulders are observed at 318, 361, 366, 374, 380, 386, 394, and $464 \mathrm{~nm}$. The absorption spectrum confirmed the presence of $\mathrm{Eu}^{3+}$.

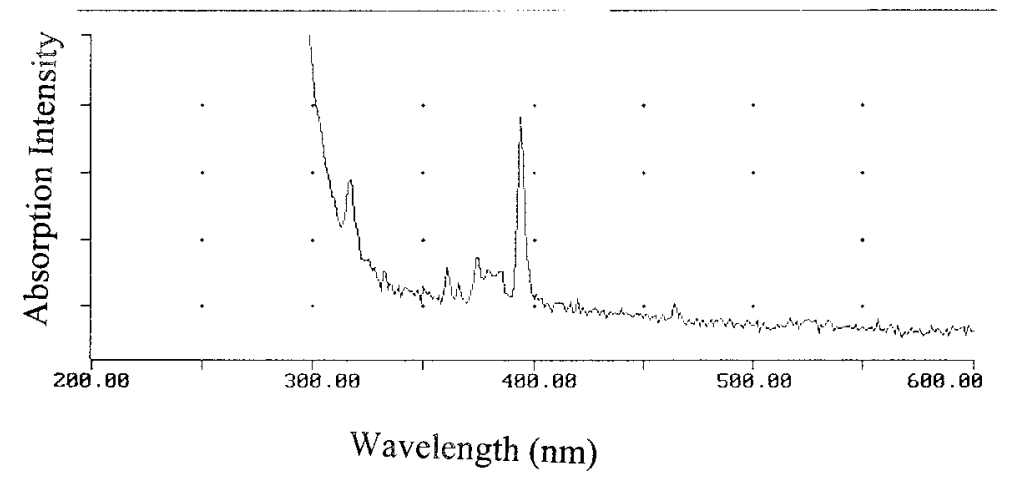

Fig. 1. Absorption spectrum of a $1 \times 10^{-3} \mathrm{M} \mathrm{Eu}^{3+}$ with excess TTT in water at room temperature. 


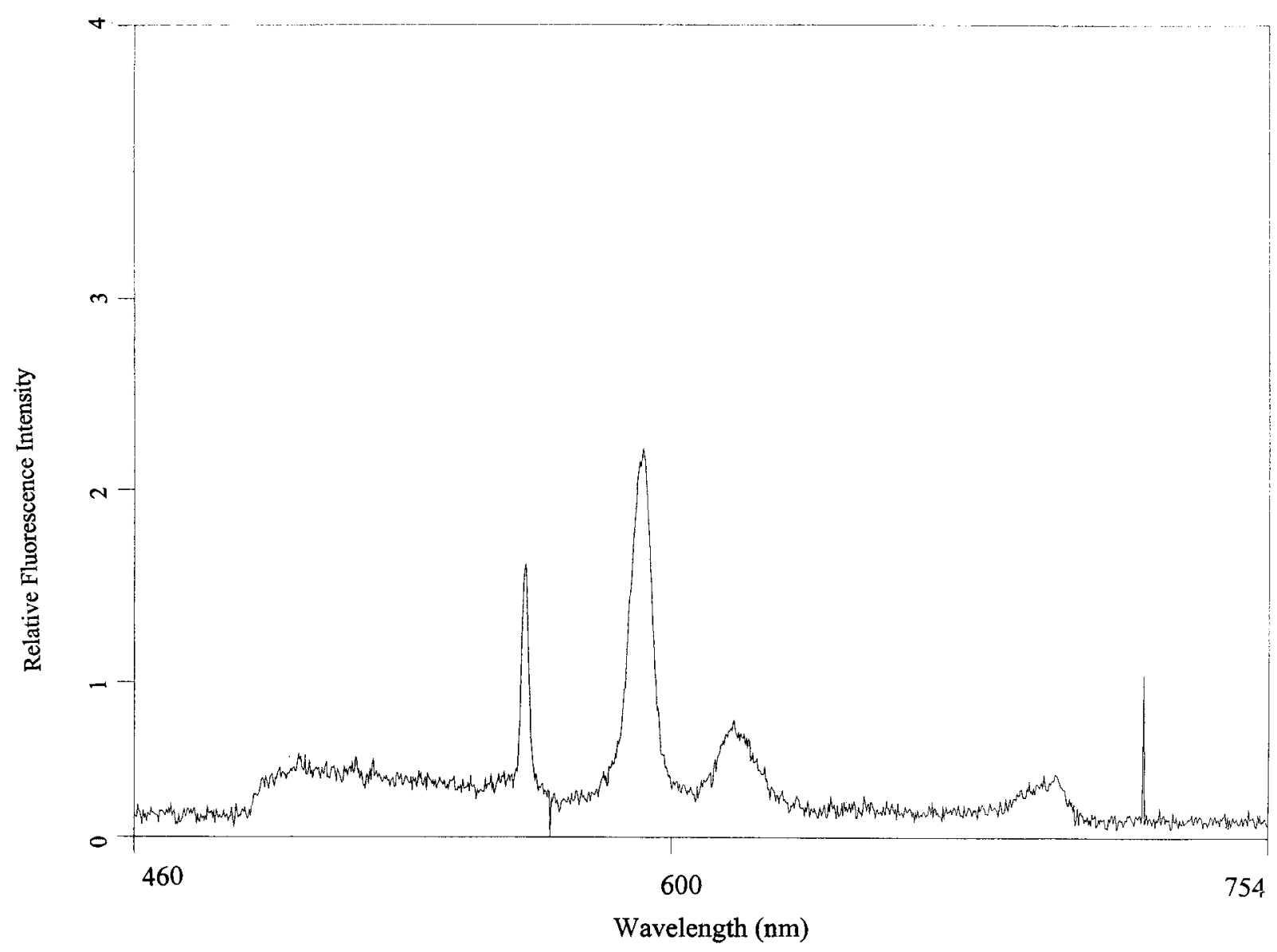

Fig. 2. Emission spectrum of a $1 \times 10^{-3} \mathrm{M} \mathrm{Eu}^{3+}\left(\mathrm{EuCl}_{3}\right)$ in water at room temperature.

\subsection{Emission spectroscopy}

Figure 2 shows the emission spectrum of a $1 \times 10^{-3} \mathrm{M} \mathrm{Eu}^{3+}$ (using $\mathrm{EuCl}_{3}$ ) in water at room temperature. Figure 3 shows the emission spectrum of a $1 \times 10^{-3} \mathrm{M} \mathrm{Eu}^{3+}$ in the presence of excess TTT in water at room temperature. The bands at 595, 617, and $698 \mathrm{~nm}$ in Fig. 2 and the bands at 592, 617, and $711 \mathrm{~nm}$ in Fig. 3 correspond to the ${ }^{5} \mathrm{D}_{0} \rightarrow{ }^{7} \mathrm{~F}_{J}$ transition of $\mathrm{Eu}^{3+}$, with $J=1,2$, and 4, respectively $[9,15]$. The sharp peaks at $\sim 560 \mathrm{~nm}$ in Figs 2 and 3 correspond to the second order of the laser excitation $(280 \mathrm{~nm})$. The line at $545 \mathrm{~nm}$ in Fig. 3 does not belong to the $\mathrm{Eu}^{3+}$ emission. Comparing Fig. 2 (unchelated $\mathrm{Eu}^{3+}$ ) to Fig. 3 (chelated $\mathrm{Eu}^{3+}$ ), a large enhancement of europium emission is observed when the ion is isolated from solvent using the bulky chelating ligand (TTT).

\subsection{Lifetime measurements}

The decay lifetime of the metastable state ${ }^{5} \mathrm{D}_{0}$ of various samples was measured at room temperature. The decay profiles of all samples are single exponentials. Table 1 summarizes the measured lifetime of all samples in both $\mathrm{H}_{2} \mathrm{O}$ and $\mathrm{D}_{2} \mathrm{O}$. 


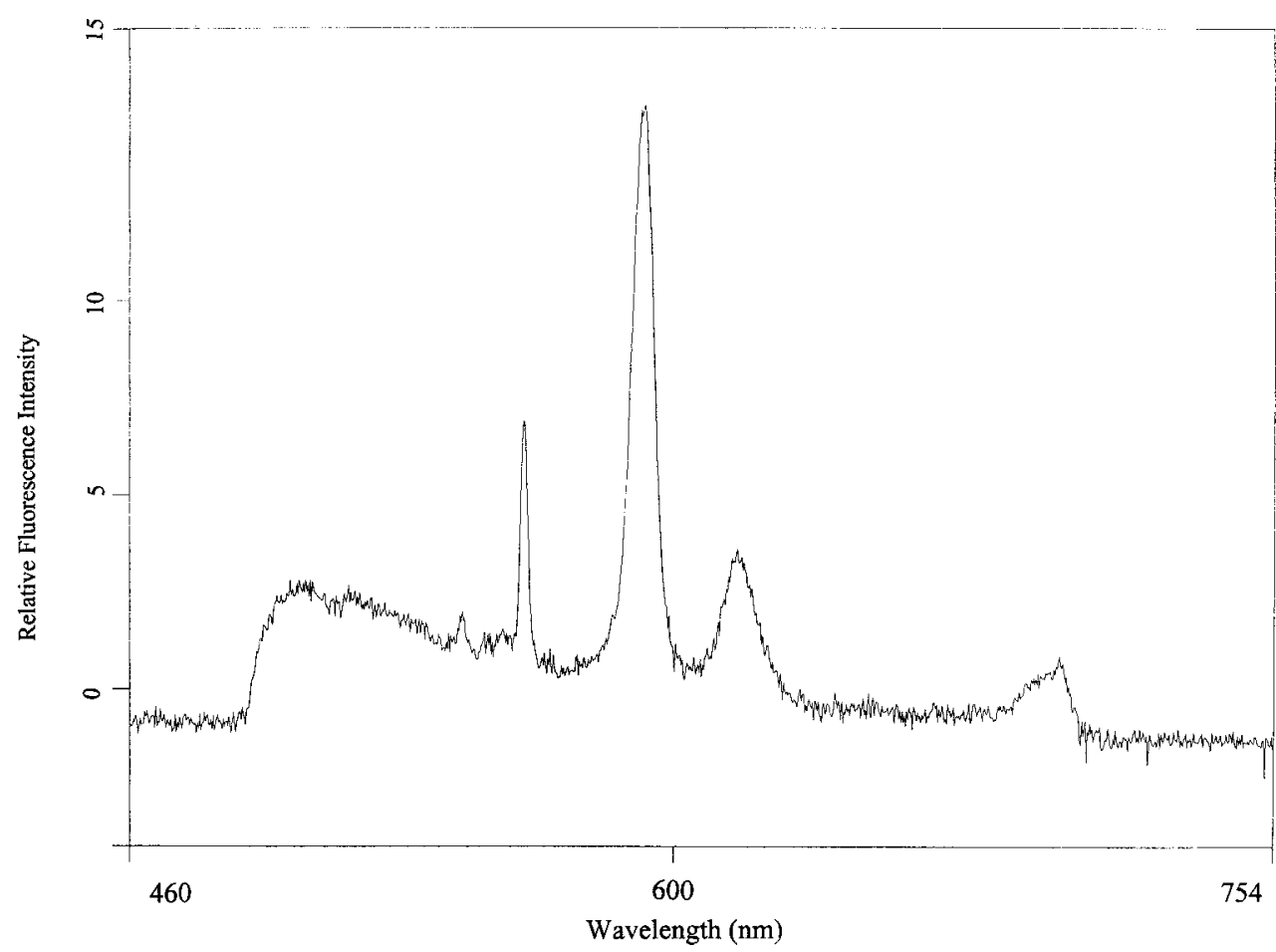

Fig. 3. Emission spectrum of a $1 \times 10^{-3} \mathrm{M} \mathrm{Eu}^{3+}$ with excess TTT in water at room temperature.

Table 1

Emission lifetimes at room temperature

\begin{tabular}{lc}
\hline Sample & Lifetime (ms) \\
\hline $\mathrm{Eu}^{3+}$ in $\mathrm{H}_{2} \mathrm{O}$ & 0.11 \\
$\mathrm{Eu}^{3+}$ in $\mathrm{D}_{2} \mathrm{O}$ & 1.12 \\
Eu-TTT in $\mathrm{H}_{2} \mathrm{O}$ & 0.60 \\
Eu-TTT in $\mathrm{D}_{2} \mathrm{O}$ & 2.39 \\
Eu-TTD in $\mathrm{H}_{2} \mathrm{O}$ & 0.63 \\
Eu-TTD in $\mathrm{D}_{2} \mathrm{O}$ & 2.47 \\
\hline
\end{tabular}

From Figs 2 and 3 and the data in Table 1, it is shown that the ratio of fluorescence intensities in Figs 2 and 3 and the ratio of the corresponding lifetimes in Table 1 are nearly the same within experimental error.

\section{Discussion}

Figure 4, shows a partial energy level diagram of $\mathrm{Eu}^{3+}$ in solution. In most cases luminescence is detected only from the ${ }^{5} \mathrm{D}_{0}$ state, irrespective of the excitation energy. Efficient nonradiative transitions among the excited states lead to a fast relaxation to the lowest one. Five narrow emission bands corresponding to ${ }^{5} \mathrm{D}_{0} \rightarrow{ }^{7} \mathrm{~F}_{J}$, where $J=0,1,2,3$, and 4 , generally characterize the europium ion [16,17]. The transitions ${ }^{5} \mathrm{D}_{0} \rightarrow{ }^{7} \mathrm{~F}_{0}$, and ${ }^{5} \mathrm{D}_{0} \rightarrow{ }^{7} \mathrm{~F}_{3}$ are too weak to be observed in Figs 2 and 3 . 


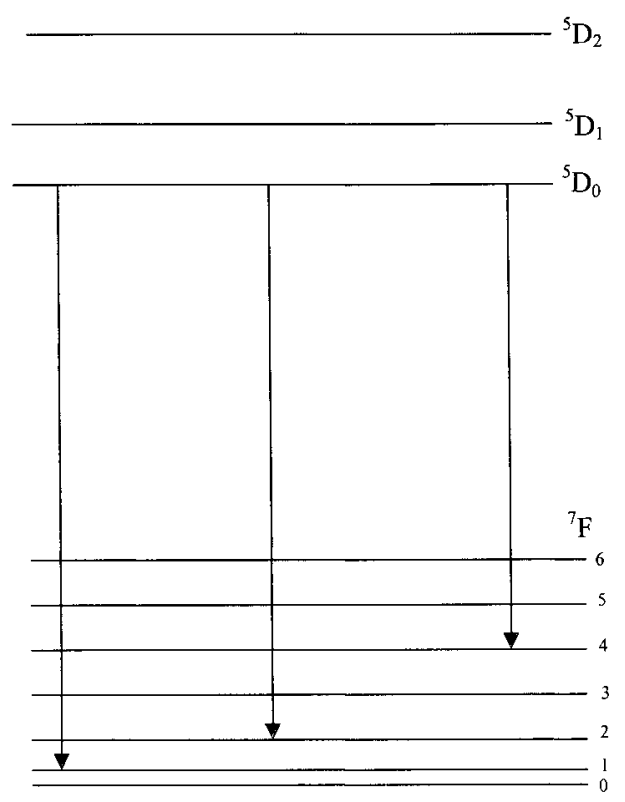

Fig. 4. Emission of $\mathrm{Eu}^{3+}$ Explained by the energy level diagram.

Concentration quenching of the luminescence of the europium ion has been the subject of many investigations $[18,19]$. Clustering of rare earth ions such as $\mathrm{Nd}^{3+}, \mathrm{Tb}^{3+}$, and $\mathrm{Eu}^{3+}$ can lead to quenching, a fact crucial for applications involving these ions. In many different media, europium decay lifetimes have been shown to decrease with increasing europium concentration. For a given $\mathrm{f}-\mathrm{f}$ transition, the energies of absorption and emission are very similar and Eu-Eu energy transfer is possible. The excitation energy can be transferred between ions until it reaches a lattice defect, which traps and dissipates the energy [1]. A second mechanism, cross relaxation, is the transfer of the excitation energy from one ion to an unexcited Eu ion, each of which dissipates its share of the energy. One of the final states in this process must decay faster compared to the initial level. Cross relaxation therefore relies critically on the relative positions of Stark levels, which depend on the host [20].

To investigate a possible concentration quenching in Eu-TTT we measured the lifetimes of $\mathrm{Eu}^{3+}$ in Eu-TTT and in Eu-TTTD crystals. The europium concentration in the later crystal is only $1 \%$ of the concentration in the former. Europium and lanthanum, having similar ionic radii, can substitute each other in a crystal and we can assume a random distribution of $\mathrm{Eu}^{3+}$ in Eu-TTTD. Absorption and emission spectra of Eu-TTT and Eu-TTTD are very similar and the observed lifetimes agree within experimental error. This result shows that in our system concentration quenching can be neglected. The Eu-Eu interaction is shielded by the presence of the bulky ligand TTT.

Weak vibronic coupling of trivalent lanthanide ion ( $\mathrm{Ln}(\mathrm{III})$ ) excited states with the $\mathrm{OH}$ oscillators of coordinated water molecules provide a facile path for radiationless deexcitation [9]. The europium emission is also quenched in the presence of hydroxyl ions and water [14,21,22]. The vibrational frequencies of $\mathrm{OH}$ groups are between 2800 and $3600 \mathrm{~cm}^{-1}$. For the deactivation of an $\mathrm{Eu}^{3+}$ ion from the ${ }^{5} \mathrm{D}_{0}$ state, several vibrations must be excited simultaneously since the energy gap between the upper state and the 
next nearest energy state is usually several times greater than the maximum energy vibrational quantum. The probability of deactivation is inversely proportional to the number of vibrational quanta that must be excited $[2,14]$. Therefore, the highest frequency vibrations, usually the $\mathrm{OH}$ stretches, play an important role in the energy transfer process. It is generally believed that the $\mathrm{O}-\mathrm{H}$ oscillators in the first coordination sphere of the metal significantly affect the luminescence decay mechanism [5].

In order to examine the effect of the high frequency $\mathrm{O}-\mathrm{H}$ modes, decay lifetime were measured in $\mathrm{H}_{2} \mathrm{O}$ as well as $\mathrm{D}_{2} \mathrm{O}$ solution. As shown in Table 1, deuterium substitution in the solvent environment markedly increase the fluorescence lifetimes ( $0.6 \mathrm{~ms}$ of Eu-TTT in water to $2.39 \mathrm{~ms}$ of Eu-TTT in $\mathrm{D}_{2} \mathrm{O}$ ). $\mathrm{O}-\mathrm{D}$ modes with their lower frequency are much less efficient in the deexcitation process. While the lifetime in $\mathrm{H}_{2} \mathrm{O}$ is reduced by a factor of about 10 compared to $\mathrm{D}_{2} \mathrm{O}$ for the free $\mathrm{Eu}^{3+}$ ion, the reduction is by a factor of 4 for Eu-TTT. This result shows that the ligand does not provide full protection of the europium ion even though all samples emit.

Table 1 shows that the difference between the rate constant (inverse of lifetime) of Eu in $\mathrm{H}_{2} \mathrm{O}$ and $\mathrm{Eu}$ in $\mathrm{D}_{2} \mathrm{O}$ is $8,000 \mathrm{~s}^{-1}$, while the difference between the rate constant of Eu-TTT in $\mathrm{H}_{2} \mathrm{O}$ and EuTTT in $\mathrm{D}_{2} \mathrm{O}$ is $1250 \mathrm{~s}^{-1}$. This means that deuterium enhancement factors are significantly lower for the Eu-TTT compared to the unchelated europium, i.e., the isotope effect does not affect the chelated and unchelated europium ions equally. This is because the coordinated shell around the unchelated $\mathrm{Eu}^{3+}$ ion is composed entirely of water molecules. Deuterium effects are, therefore, maximum in this system. In Eu-TTT system, Eu-TTT exists in aqueous solution as a chelated species and thus it has fewer water molecules in the first coordination sphere. Deuterium effects would, therefore, be expected to be less and this is borne out by the experimental results. This further proves that the ligand does not provide full protection of the Eu ion.

Table 1 also shows that the chelate effect on $\mathrm{Eu}^{3+}$ fluorescence lifetime is solvent dependent. Our experimental data (Table 1) shows that the difference between the rate constant (inverse of lifetime) of $\mathrm{Eu}$ in $\mathrm{H}_{2} \mathrm{O}$ and Eu-TTT in $\mathrm{H}_{2} \mathrm{O}$ is $7400 \mathrm{~s}^{-1}$, while the difference between the rate constant of Eu in $\mathrm{D}_{2} \mathrm{O}$ and Eu-TTT in $\mathrm{D}_{2} \mathrm{O}$ is $470 \mathrm{~s}^{-1}$. TTT is shown to alter the fluorescence lifetime of $\mathrm{Eu}^{3+}$ ion in water more that it does in the deuterated solvent. In $\mathrm{H}_{2} \mathrm{O}$, TTT replaces many water molecules surrounding the $\mathrm{Eu}^{3+}$ ion and thus has a large enhancement (about 5 times) of lifetime $(0.11$ to $0.6 \mathrm{~ms})$. In $\mathrm{D}_{2} \mathrm{O}$, the lifetime is increased by a factor of 2 (1.12 to 2.39) in the case of unchelated (naked) europium compared to Eu-TTT. The enhancement of lifetime in $\mathrm{D}_{2} \mathrm{O}$ is probably due to the change of asymmetry around the $\mathrm{Eu}^{3+}[14]$.

In summary the organic ligand TTT was used to chelate the $\mathrm{Eu}^{3+}$ ion in solution and protect it from the deactivating hydroxyl groups. TTT turns out to partially protect the $\mathrm{Eu}^{3+}$ ion from the local surroundings and reduce radiationless deactivation of the excited state. By comparing Eu-TTT with Eu-TTTD, it was shown that concentration quenching is not important in this system. Water quenching, however, is still significant as shown by the difference between the lifetimes of the europium emission in $\mathrm{H}_{2} \mathrm{O}$ and $\mathrm{D}_{2} \mathrm{O}$ solutions.

\section{Experimental}

The chelate Eu-TTT used for this study is an europium chelate containing the ligand TTT (Aldrich). Two different materials were prepared: pure Eu-TTT crystals and Eu-TTTD crystals containing 1\% EuTTT doped in the corresponding lanthanum compound $\left(\mathrm{Eu}_{0.01} \mathrm{La}_{0.99}-\mathrm{TTT}\right)$.

$\mathrm{Eu}$-TTT was prepared by mixing an aqueous europium chloride solution $\left(\mathrm{EuCl}_{3}\right.$, Aldrich) and a warm aqueous solution of the ligand TTT followed by dropwise addition of $1.0 \mathrm{M}$ sodium hydroxide until a 
$\mathrm{pH}$ of about 8 was obtained. Slow evaporation over a period of several days under ambient conditions resulted in white crystals.

For the preparation of the Eu-TTTD crystals, the above method was modified in the following manner: before adding the TTT solution, a solution of $\mathrm{La}_{2} \mathrm{O}_{3}$ (Aldrich) in dilute nitric acid was added to the aqueous solution of $\mathrm{EuCl}_{3}$ in a 99: 1 lanthanum to europium mole ratio. Solutions of Eu-TTT, Eu-TTTD, and $\mathrm{EuCl}_{3}$ in water or deuterium oxide were used for the spectroscopic studies. In all cases the ligand was added in excess to make sure that all the europium ions are chelated.

The luminescence spectra and lifetimes of $\mathrm{Eu}^{3+}$ were recorded by using a dye laser pumped by a Q-switched ND:YAG laser (Spectron INC, SL800,) pumped dye laser (Lambda Physik. M2220). The dye laser of Rhodamine $6 \mathrm{G}$ was frequency doubled with a second harmonic generation module to generate the $280 \mathrm{~nm}$ light used for excitation. The emission spectra were scanned 500 times and averaged in an optical multichannel analyzer (EG\&G, OMA 1461 with CCD detection). The transient luminescent signal was focused onto a monochromator (spex, 1404) and detected by a Hamamatzu, R928 photomultiplier tube. A fast signal processor (EG\&G, model 4400) in combination with a boxcar averager was used to measure the emission decay time. Absorption spectra were recorded on a Cary $3 \mathrm{E}$ UV-vis spectrophotometer.

\section{Acknowledgment}

The Author is grateful to Dr. Taesam Kim at the Korea Institute of Geology, Mining and Materials (30, Kajung-Dong, Yusung-Ku, Taejon, Korea 305-350) for running the absorption, emission and life time measurements.

\section{References}

[1] M.J. Lochhead and L.K. Barry, Better ceramics through chemistry VI, in: Proceedings of the Materials Research Society Symposium 346, A.K. Cheetham, C.J. Brinker, M.L. Mecartney and C. Sanchez, eds, Materials research Society, Pittsburgh, PA, 1994, p. 745.

[2] D.C. Lia, B. Dunn and J.I. Zink, Inorg. Chem. 35 (1996), 2152.

[3] N. Koslova, B. Viana and C. Sanchez, J. Mater. Chem. 3 (1993), 111.

[4] B. Viana, N. Koslova, P. Achehoug and C. Sanchez, J. Mater. Chem. 5 (1995), 711.

[5] M.R. Lowell and E.T. Knobbe, Chem. Mater. 5 (1993), 1697.

[6] E.J. Popse and J.D. Mackenzie, J. Non-Crys. Solids 106 (1988), 236.

[7] T. Fujiyama, T. Yokoyama, M. Hori and M. Sasaki, J. Non-Cryst. Solids 135 (1991), 198.

[8] I. Thomas, S. Payne and G.D. Wilke, J. Non-Cryst. Solids 152 (1992), 183.

[9] W.D. Horrocks and D.R. Sudnick, J. Am. Chem. Soc. 101 (1979), 334.

[10] K.L. Bray and M.J. Lohhead, J. Non-Cryst. Solids 170 (1994), 143.

[11] S.L. Wu and W.D. Horrocks, Jr., Analyt. Chem. 68 (1996), 394.

[12] G. Blasse, in: Physics and Chemistry of Rare Earths., Vol. 3, K.A. Gschneider and L. Eyring, eds, North-Holland Publishing Company, New York, 1979, p. 275.

[13] D.W. Hairston, Chemical Engineering (January 1997), 57.

[14] J.L. Kropp and M.W. Windsor, J. Chem. Phys. 42 (1965), 1599.

[15] G. Blasse, G. Dirksen and A. Meijerink, Chem. Phys. Lett. 167 (1990), 41

[16] Y. Hass and G. Stein, Chem. Phys. Lett. 366 (1971), 3668.

[17] G. Carturan, R. Campostrini, M. Ferrari, M. Montagana and O.J. Pilla, J. Mater. Res. 7 (1992), 745.

[18] K. Aria, H. Namikawa, K. Kumata, T. Honda, Y. Ishii and T. Handa, J. Appl. Phys. 59 (1986), 3430.

[19] I.M. Thomas, S.A. Payne and G.D. Wilke, J. Non-Cryst. Solids 151 (1992), 183.

[20] G. Blasse, in: Physics and Chemistry of Rare Earths, K.A. Gschneider and L. Eyring, eds, North-Holland Publishing Company, New York, 1979, p. 237.

[21] Y. Hass and G. Stein, J. Phys. Chem. 75 (1971), 3677.

[22] G. Stein and E. Wurzberg, J. Chem. Phys. 62 (1975), 208. 


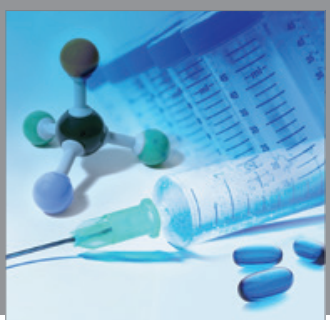

International Journal of

Medicinal Chemistry

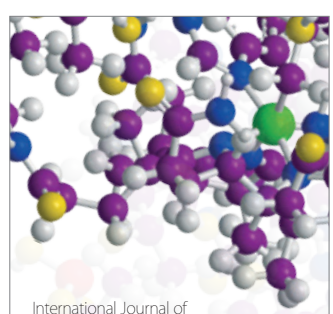

Carbohydrate Chemistry

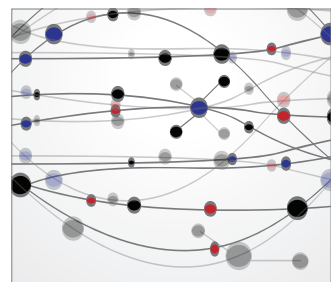

The Scientific World Journal
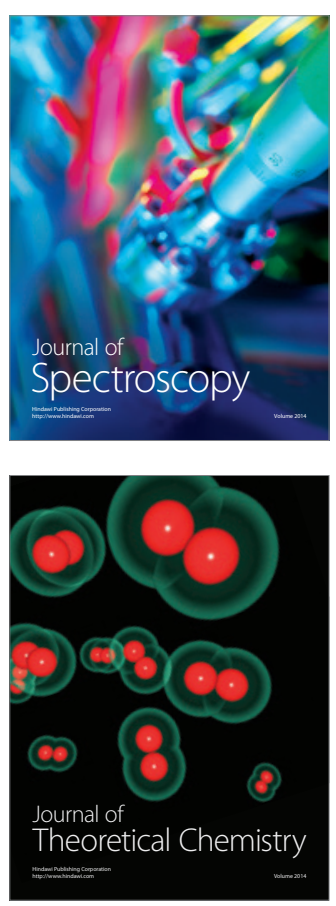
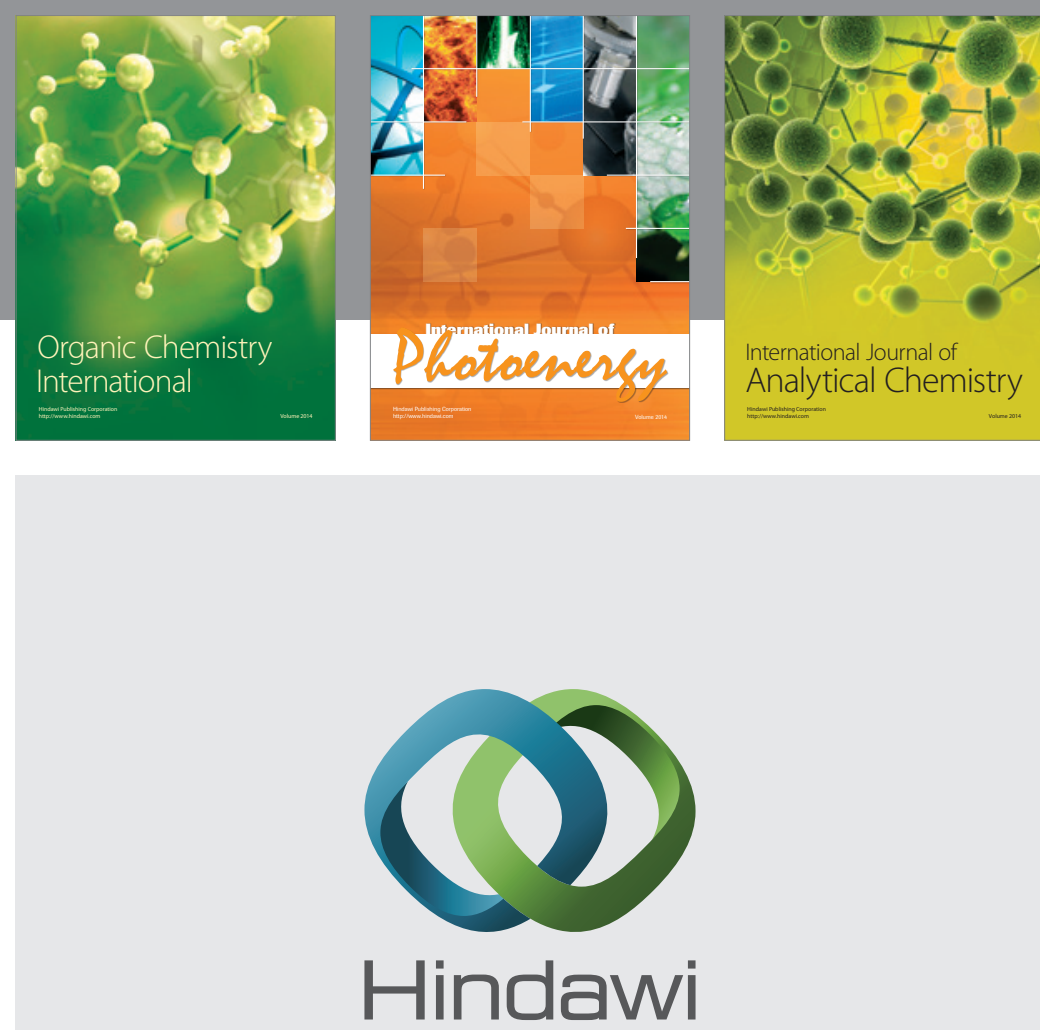

Submit your manuscripts at

http://www.hindawi.com
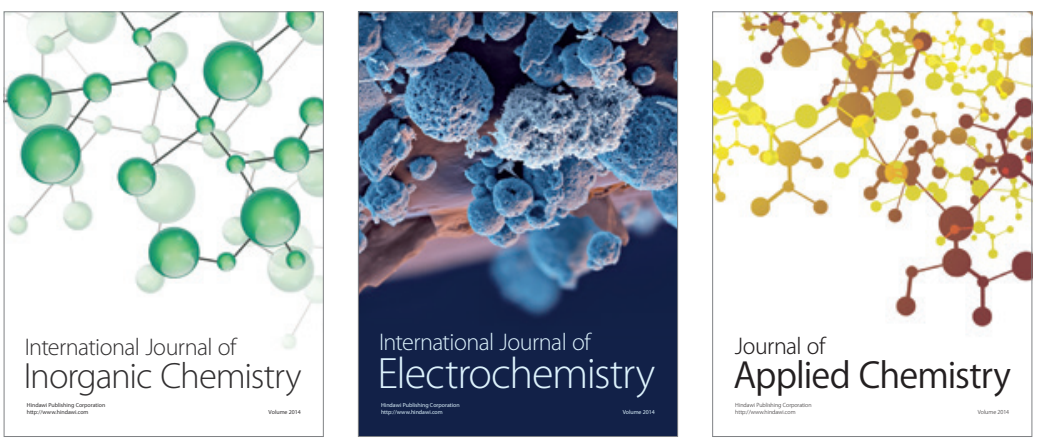

Journal of

Applied Chemistry
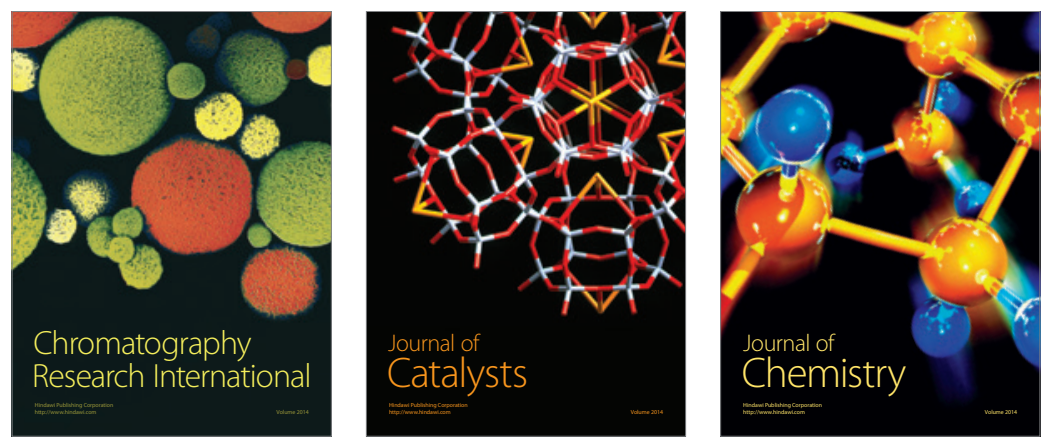
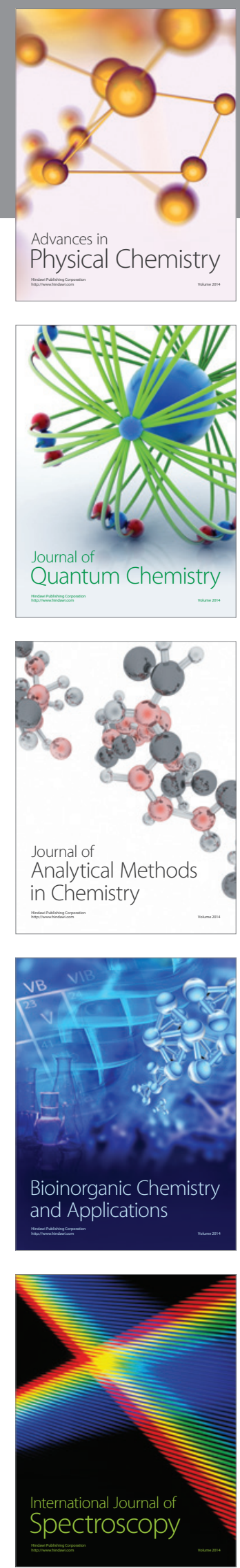